\title{
Enfermeiros de urgência e emergência no atendimento à pacientes com transtornos psiquiátricos
}

\author{
Nurses in the emergency and psychiatric services
}

\section{Enfermeras en los servicios de emergencia y psiquiátricos}

Diorges Boone da Silva ${ }^{1 *}$, Antonia Eide de Souza do Carmo², Edney Bentes de Oliveira², Emelly Cristina Silva Rodrigues², Glória Evelly Nogueira Bezerra², Pablo da Conceição Castro³.

\section{RESUMO}

Objetivo: Descrever a atuação dos profissionais de enfermagem em urgência e emergência nos atendimentos a pacientes com transtornos psiquiátricos. Métodos: Estudo de revisão integrativa. A pesquisa foi concretizada por meio da Biblioteca Virtual em Saúde (BVS), utilizando critérios de inclusão e exclusão das publicações, selecionando artigos através dos Descritores em Saúde (DeCS) publicados a partir do ano de 2015. Resultados: Foram encontrados um total de 2.592 achados, destes, 20 atenderam aos critérios elencados para os resultados. Após a análise, escolheu-se duas categorias com 10 artigos cada, sendo a primeira: Os procedimentos de enfermagem no atendimento inicial dos pacientes com transtornos mentais, $\mathrm{e}$ a segunda categoria: A formação e a experiência profissional. Considerações finais: Os transtornos psiquiátricos são bastante prevalentes, e as situações de emergências psiquiátricas são rotineiras. Portanto, a equipe de enfermagem que compõem este departamento precisa compreender o processo dos transtornos mentais e como atuar em diferentes circunstâncias, além da prática é relevante todo conhecimento teórico que possa ser instituída por meio de educação permanente.

Palavras-chave: Transtornos psiquiátricos, Saúde mental, Enfermagem, Emergência.

\begin{abstract}
Objective: To describe the performance of nursing professionals in urgency and emergency care in patients with psychiatric disorders. Methods: Study of integrative revision. The research was carried out through the Virtual Health Library (VHL), using inclusion and exclusion criteria of publications, selecting articles through the Health Descriptors (DeCS) published from 2015. Results: A total of 2,592 findings were found, 20 of which met the criteria listed for the results. After the analysis, two categories were chosen with 10 articles each, the first: Nursing procedures in the initial care of patients with mental disorders, and the second category: Training and professional experience. Final considerations: Psychiatric disorders are quite prevalent, and psychiatric emergency situations are routine. Therefore, the nursing team that compose this department needs to understand the process of mental disorders and how to act in different circumstances, beyond the practice is relevant all theoretical knowledge that can be instituted through permanent education.
\end{abstract}

Keywords: Psychiatric disorders, Mental health, Nursing, Emergency.

${ }^{1}$ Centro Universitário Fametro (CEUNI-FAMETRO), Manaus - AM. *E-mail: diorges248@gmail.com

${ }^{2}$ Centro Universitário do Norte (UNINORTE), Manaus - AM.

${ }^{3}$ Faculdade Estácio do Amazonas (Estácio Amazonas), Manaus - AM.

SUBMETIDO EM: 10/2020

ACEITO EM: 11/2020

PUBLICADO EM: 2/2021 
RESUMEN

Objetivo: Describir el desempeño de los profesionales de la enfermería en la atención de urgencia y emergencia en pacientes con trastornos psiquiátricos. Métodos: Estudio de revisión integradora. La investigación se llevó a cabo a través de la Biblioteca Virtual de Salud (BVS), utilizando criterios de inclusión y exclusión de publicaciones, seleccionando los artículos a través de los Descriptores de Salud (DeCS) publicados a partir de 2015. Resultados: Se encontraron un total de 2.592 hallazgos, 20 de los cuales cumplían con los criterios listados para los resultados. Tras el análisis, se eligieron dos categorías con 10 artículos cada una, la primera de ellas: Procedimientos de enfermería en la atención inicial de pacientes con trastornos mentales, y la segunda categoría: Formación y experiencia profesional. Consideraciones finales: Los trastornos psiquiátricos son bastante frecuentes, y las situaciones de emergencia psiquiátrica son rutinarias. Por lo tanto, el equipo de enfermería que compone este departamento necesita comprender el proceso de los trastornos mentales y cómo actuar en diferentes circunstancias, además de la práctica, todos los conocimientos teóricos que se pueden instituir a través de la educación permanente son relevantes.

Palabras clave: Trastornos psiquiátricos, Salud mental, Enfermería, Emergencia.

\section{INTRODUÇÃO}

O final da década de 70, foi marcado por grandes transformações no contexto da saúde mental no Brasil, momento este em que a reforma psiquiátrica surgia, possibilitando mudanças sociopolíticas, no modelo de assistência e serviços de saúde pela superação das violências manicomiais, bem como instituir direitos as pessoas com transtornos psiquiátricos (BRASIL, 2005).

Os avanços na saúde mental foram significativos para uma melhor abordagem e conduta, salientando que ainda é um assunto pouco difundido na área de atuação da equipe de enfermagem em urgência e emergência, podendo ser classificado como um problema de saúde pública. Os transtornos psiquiátricos podem ocorrer quando existe uma perturbação do funcionamento do sistema nervoso central (SNC) ou em sua iminência, sendo estas alterações agudas do pensamento, comportamento ou das relações sociais, necessitando em alguns casos de intervenção (BRITO AFG, et al., 2017).

Segundo a Organização Mundial da Saúde (OMS), os transtornos mentais acometem mais de 450 milhões de pessoas e respondem por $12,3 \%$ das causas de enfermidades e invalidez. Nos Estados Unidos, estimase que a prevalência de transtornos mentais graves na população geral seja de $4,2 \%$. No Brasil, foram encontrados altos índices de transtornos mentais na população adulta, entre 20 a 56\%, com destaque para populações específicas, como mulheres e trabalhadores, estes números podem somar um total de 562 milhões em 2020 (OMS, 2012; COSTA JM, et al., 2019).

Desta forma, o enfermeiro que atua nas urgências e emergências psiquiátricas, deve usar a percepção, observação e criar uma estratégia para o campo de ação com tomada decisão de forma planejada, visto que está área ainda é conflituosa, tornando-se indispensável à intervenção imediata de uma equipe multiprofissional treinada, para evitar maiores prejuízos à saúde do indivíduo, ou de eliminar possíveis riscos à sua vida ou à de terceiros (DEU-BEM CM, et al., 2017; VARGAS D, et al., 2017; COSTA JM, et al., 2019).

De acordo com Paes MR e Maftum MA (2013), mesmo sendo de grande importância a existência de um atendimento de qualidade as pessoas que necessitam de atenção psiquiátrica de emergência, ainda existe uma grande dificuldade por parte da equipe de enfermagem no que tange esta abordagem, encontrando-se uma falta de conhecimento para tal, assim como comunicação e inexperiência.

A luta pela reforma psiquiátrica, trouxe a desinstitucionalização e a inclusão social da pessoa com transtorno mental, assim, houve um aumento das redes de atenção psicossocial (RAPS) para atender e tratar estas ocorrências. De tal forma, os quadros de emergências são encaminhados para unidades diversificadas, outros para aquelas com especialidades e um número reduzido para as instituições que realizam atendimento nos finais de semanas feriados e atendimento 24h. OS Centro de Atenção Psicossocial (CAPS) de urgências e emergências psiquiátricas são unidades mistas que dispõem de um atendimento para situações graves e funcionam 24h (OHARA R, et al., 2010; CAVEIRÃO C, et al., 2015). 
A disseminação de estudos no que diz respeito ao conhecimento sobre as urgências e emergências psiquiátricas no contexto da enfermagem, é de suma importância, trazendo um olhar equânime para o atendimento e resgate destas pessoas em situação de riscos, reduzindo o sofrimento destes e impedindo qualquer dano a sua integridade, fortalecendo o alicerce dos protocolos e das políticas públicas de saúde mental (CAVEIRÃO C, et al., 2015).

As pessoas que sofrem de transtornos mentais, podem estar presentes nos mais diferentes tipos de serviços de atendimento à saúde, sendo necessário que equipe de enfermagem seja capacitada no que diz respeito ao atendimento à saúde mental, pois esta clientela permeia as diversas áreas de atuação da enfermagem, sendo que, em qualquer que seja o cenário, as emergências psiquiátricas podem ocorrer, necessitando de medidas eficazes no atendimento (CRISTIANO CC, et al., 2015).

Por tanto emergiu-se o intuito de realizar o presente estudo, na tentativa de aprofundar um olhar para este público marginalizado a décadas pela sociedade, família e em muitos dos casos pelos próprios profissionais de saúde. Assim, esta proposta traz um olhar para as urgências e emergências psiquiátricas, objetivando descrever a atuação dos profissionais de enfermagem da urgência e emergência nos atendimentos aos pacientes com transtornos psiquiátricos.

\section{MÉTODOS}

O presente artigo traz um estudo bibliográfico descritivo de revisão integrativa da literatura. O estudo foi realizado por meio de bases de dados bibliográficas nacionais e internacionais, estes cientificamente válidas, por meio da BVS, elegendo artigos através dos DeCS: Transtornos psiquiátricos, Saúde mental, Enfermagem, Emergência, foi utilizada a expressão "AND" para uma melhor efetividade de achados, para melhor captar os artigos, utilizou-se o método de filtragem de idiomas da língua portuguesa e inglesa, disponíveis na Biblioteca Científica Eletrônica Online (SciELO), Literatura Latino-Americano e do Caribe (LILACS) e Medical Literature Analysis and Retrieval System Online (MEDLINE).

No processo de seleção dos artigos, na tentativa de aprimorar a abordagem do conteúdo, bem como sua atualização, considerou-se aqueles que correspondiam aos critérios de inclusão definindo: Artigos que contextualizem a ideia da proposta, os DeCS nas buscas realizadas, e artigos publicados a partir do ano de 2015.

No que se refere a exclusão dos artigos, foi definido a partir de: Artigos repetidos em demais bases de busca; não se referirem a adultos; artigos que fujam da temática central da pesquisa; estudos sobre outras patologias e ausência de acesso ao texto na íntegra.

Os estudos foram sendo considerados elegíveis de acordo com seu enquadramento nos critérios, seguidos de avaliação por meio do título, resumo, leitura na integra e desta forma sendo selecionados para a revisão.

Posteriormente foi realizada uma leitura minuciosa dos artigos que passaram pelo processo de seleção, o que possibilitou a discussão dos dados encontrados. Os achados foram tratados de forma descritiva através de percentuais qualitativos, referentes à caracterização dos estudos.

A análise dos dados originou um eixo temático denominado: visão geral dos atendimentos psiquiátricos realizados nos serviços de urgência e emergência. Realizou-se a construção de quadro e tabelas, sobre os dados analisados, os quais foram discutidos com base na literatura pertinente para melhorar a compreensão. A triagem foi realizada pelos integrantes da pesquisa, desta forma chegando ao resultado e desenho do estudo, onde todos realizaram o mesmo processo de inclusão e exclusão dos artigos encontrados.

\section{RESULTADOS E DISCUSSÃO}

Este estudo aborda uma descrição interpretativa dos achados na literatura, por meio dos DeCS e os critérios citados para busca e seleção, foram encontrados 20 artigos na Scielo, 2.592 na Medline e 216 na Lilacs, totalizando 2.828 achados, destes, apenas 20 artigos atenderam aos critérios para elencar os resultados. 
No que concerne a temática proposta, a literatura dispõem de vários aspectos, assim como diferentes tipos de abordagem, foi possível identificar que apesar das bases de dados dispor de meios que gerem dados para a construção de um pensamento acerca do estudo, a mesma necessita de amplo aprofundamento voltados para a área das urgências e emergências psiquiátricas, assim como uma maior observância de como os profissionais abordam estes paciente, demonstra os achados que foram de suma importância para a discussão do artigo (Quadro 1).

Quadro 1 - Realização da busca de artigos através dos DeCS e seleção para os resultados e discussão.

\begin{tabular}{|c|c|c|c|c|c|c|}
\hline $\begin{array}{l}\text { Base de } \\
\text { Dados }\end{array}$ & DeCS & $\begin{array}{l}\text { Número de } \\
\text { Referências } \\
\text { obtidas }\end{array}$ & $\begin{array}{l}\text { Seleção através } \\
\text { dos critérios de } \\
\text { elegibilidade e } \\
\text { inelegibilidade }\end{array}$ & $\begin{array}{c}\text { Resumos } \\
\text { Analisados } \\
\text { por meio } \\
\text { dos Títulos }\end{array}$ & $\begin{array}{l}\text { Analisados } \\
\text { na integra }\end{array}$ & $\begin{array}{l}\text { Selecionados } \\
\text { para Revisão }\end{array}$ \\
\hline \multirow{3}{*}{ SciELO } & $\begin{array}{c}\text { Transtornos } \\
\text { psiquiátricos } \\
\text { and } \\
\text { Emergência }\end{array}$ & 5 & $x_{0}$ & 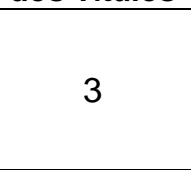 & 3 & 3 \\
\hline & $\begin{array}{l}\text { Saúde mental } \\
\text { and } \\
\text { Enfermagem }\end{array}$ & 4 & 1 & 1 & 1 & 1 \\
\hline & $\begin{array}{l}\text { Transtornos } \\
\text { psiquiátricos } \\
\text { and } \\
\text { Enfermagem }\end{array}$ & 11 & 7 & 5 & 4 & 3 \\
\hline \multirow{3}{*}{ MEDLINE } & $\begin{array}{c}\text { Transtornos } \\
\text { psiquiátricos } \\
\text { and } \\
\text { Emergência } \\
\end{array}$ & 359 & 72 & 30 & 4 & 3 \\
\hline & $\begin{array}{l}\text { Saúde mental } \\
\text { and } \\
\text { Enfermagem }\end{array}$ & 22 & 15 & 1 & 1 & 1 \\
\hline & $\begin{array}{c}\text { Transtornos } \\
\text { psiquiátricos } \\
\text { and } \\
\text { Enfermagem }\end{array}$ & 2.211 & 995 & 15 & 6 & 3 \\
\hline \multirow{3}{*}{ LILACS } & $\begin{array}{c}\text { Transtornos } \\
\text { psiquiátricos } \\
\text { and } \\
\text { Emergência }\end{array}$ & 22 & 10 & 8 & 5 & 3 \\
\hline & $\begin{array}{l}\text { Saúde mental } \\
\text { and } \\
\text { Enfermagem }\end{array}$ & 54 & 8 & 4 & 2 & 1 \\
\hline & $\begin{array}{l}\text { Transtornos } \\
\text { psiquiátricos } \\
\text { and } \\
\text { Enfermagem }\end{array}$ & 140 & 48 & 16 & 12 & 2 \\
\hline
\end{tabular}

Fonte: Silva DB, et al., 2020.

Após a análise, escolheu-se duas categorias, sendo a primeira: Os procedimentos de enfermagem no atendimento inicial dos pacientes com transtornos mentais, selecionando 10 artigos que abordam a matriz de síntese, e a segunda categoria; A formação e a experiência profissional, com 10 artigos.

\section{Os procedimentos de enfermagem no atendimento à pacientes com transtornos psiquiátricos}

Desde o advento da Reforma Psiquiátrica, os serviços de urgência e emergência psiquiátrica vêm sendo negligenciados, pela inobservância de suas características quanto ao número de consultas realizadas, principais psicopatologias, idade e sexo com maior prevalência entre a população, tornando-se importante avaliar o perfil dos usuários. Compreender estes aspectos reforça a existência da necessidade de que medidas mais efetivas e eficientes sejam impostas nos serviços extra-hospitalares (LIMA ICS e GUIMARÃES A, 2015). 
O transtorno pode ser caracterizado como uma situação de emergência, onde uma pessoa enfrenta dificuldades cotidianas, apresentando um desequilíbrio emocional no qual as pessoas não conseguem lidar com tal problema, desta forma estas desordens mentais, podem fazer com que a equipe de enfermagem desenvolva sentimentos relacionados ao medo, vulnerabilidade e insegurança, dificultando $o$ atendimento primário (FERNANDES MA, et al., 2016; PIMENTA FJNA e BARROS MMA, 2019).

Neste atendimento inicial, é recomendado que o Serviço de Atendimento Móvel de Urgência (SAMU), realize os primeiros procedimentos e encaminhe a pessoa em crise a um ponto da rede de urgência e emergência com especialidade para tal.

Os atendimentos mais recorrentes são: psicoses, tentativa de suicídio, depressões e síndromes cerebrais orgânicas, porém na maioria dos casos, os profissionais não dispõem de capacitação para este manejo, o que dificulta a atenção a este público (ALMEIDA AB, et al., 2015; BONFADA D, et al., 2015). Observa-se que, é importante compreender a situação da pessoa em crise psiquiátrica, para que se utilize de recursos eficazes e que estes sejam cabíveis para cada atendimento ou intervenção (Quadro 2).

Quadro 2 - Classificação quanto aos tipos de manejos pela equipe de urgência e emergência.

\begin{tabular}{|c|l|}
\hline \multicolumn{1}{|c|}{ Tipos } & \multicolumn{1}{c|}{ Descrição } \\
\hline Manejo atitudinal & $\begin{array}{l}\text { Estar vigilante e pronto para a ação, evitar movimentos bruscos e confronto } \\
\text { direto, respeitar o espaço físico, manter-se atento à linguagem não verbal, } \\
\text { reduzir os estímulos, afastar fatores avaliados como estressores ou } \\
\text { desestabilizadores. }\end{array}$ \\
\hline Manejo verbal & $\begin{array}{l}\text { Utilizar linguagem simples, clara e concreta, evitar elevar o tom de voz, } \\
\text { estabelecer limites, evitar confronto direto, estimular expressão verbal de } \\
\text { sentimentos, repetir as últimas ideias do cliente, assegurar que você } \\
\text { pretende ajuda-lo a controlar os próprios impulsos, evitar ceder a testes, } \\
\text { desafios e manipulações, bem como evitar promessas, ameaças, opiniões } \\
\text { pessoais e explicar as condutas terapêuticas. }\end{array}$ \\
\hline A contenção & $\begin{array}{l}\text { Utilização de meios físicos ou farmacológicos para impedir comportamentos } \\
\text { destrutivos. No Brasil, as contenções físicas e químicas devem ser } \\
\text { prescritas por médicos, devendo esta ser empregada apenas se outros } \\
\text { métodos não tiverem efeito. }\end{array}$ \\
\hline Contenção física & $\begin{array}{l}\text { Utilização de fármacos para impedir comportamentos destrutivos. A via oral } \\
\text { é preferível para a aplicação da contenção química, sempre que possível e } \\
\text { que o paciente esteja colaborativo. Em caso de paciente não cooperativo, o } \\
\text { medicamento pode ser administrado por via intramuscular. } \\
\text { evitar comportamentos destrutivos, objetivando controlar o indivíduo para } \\
\text { preservar sua própria segurança e integridade ou de terceiros. }\end{array}$ \\
\hline Conten
\end{tabular}

Fonte: Silva DB, et al., 2020.

Podemos observa que existem medidas a serem escolhidas de acordo com a situação em que a pessoa com transtorno psíquico se encontra, viabilizando uma melhor abordagem para manter a integridade física da 
mesma, dos profissionais, familiares e demais, assim possibilitando um acolhimento desde o primeiro contato até um direcionamento destes para uma unidade que ofertara uma atenção especializada.

Em qualquer tipo de atendimento pela equipe de urgência e emergência, é trivial a avaliação da cena, quando não houver risco para a equipe, estabelecer um diálogo para avaliar sinais e sintomas, geralmente pessoas em surtos confundem a realidade, e neste momento o profissional pode utilizar etapas descritas na tabela supracitada, assim é possível seguir uma assistência de acordo com a necessidade ou grau de periculosidade, se houver perigo é importante o auxílio da polícia, nos casos em que o paciente possuir algum tipo de arma. Sendo necessário toda e qualquer tomada de decisão ser repassada ou acatada pela regulação (ALMEIDA AB, et al., 2015).

No que se refere à contenção mecânica, sua utilização é indicada pelo Conselho Federal de Medicina na Resolução $n^{\circ} 1.598 / 2000$, e que sua prescrição seja realizada pelo profissional médico conforme descrito no art. 11. Para o Conselho Federal de Enfermagem, na resolução $n^{\circ} 427 / 2012$, são normatizados os procedimentos para os cuidados no emprego de contenção mecânica (KONDO EH, et al., 2011; CAVEIRÃO C, et al., 2015).

Outro estudo realizado por Bonfada D e Guimarães J (2015), demonstra que muitas condutas realizadas ainda se associam à prática manicomial, pois os profissionais faziam o uso da força coercitiva, sendo exercida até com o auxílio de policiais e sem indicação médica para tal, visto que quando a mesma não está indicada pode trazer danos físicos, como necrose, trombos, fraturas, aspiração, asfixia e aumento dos problemas psíquicos para os pacientes (PIMENTA FJNA, et al., 2019).

Segundo Gonçalves KG, et al. (2019) dos atendimentos de urgências psiquiátricas, cerca de $10 \%$ a $45 \%$ chegam a apresentar comportamento agressivo, agitado ou ameaçador. Os homens em sua maioria apresentam prevalência de transtornos advindos do uso de substâncias psicoativas o que ocasiona as crises.

De acordo Andrade ACS, et al. (2016), muitos serviços de atendimento extra-hospitalar, no que diz respeito a saúde mental, apresentam insuficiência em diversos aspectos, desde o profissional que faz a captação, aos serviços hospitalares que prestam os cuidados. Pois, o que se observa nos dias atuais na sociedade em geral, é uma negatividade quanto aos pacientes com transtornos mentais, sendo vistos como incapazes, agressivos, perigosos, assim os deixando as margens da sociedade em diversos aspectos, devido aos entraves sociais, emocionais e culturais a implantação de um serviço extra-hospitalar se torna a cada dia mais difícil (BASTOS F, et al., 2018).

Salienta-se que, o diagnóstico realizado durante o atendimento tem importante implicação terapêutica, tornando-se decisivo para a tomada de decisão na escolha do manejo e conduta, entretanto os diagnósticos de transtornos psiquiátricos nas urgências e emergências são de baixa qualidade, sendo pouco eficazes e as vezes resulta na utilização de medidas inadequadas ou sem a devida necessidade (LOPES MCBT, et al., 2018).

Um atendimento com grande teor de agilidade no manejo do paciente, se torna essencial para um adequado funcionamento dos serviços de emergências, contudo, esta não é uma realidade para os profissionais neste tipo de serviço. Em geral, as urgências e emergência psiquiátrica têm pouca disponibilidade de leitos para melhor observação e acompanhamento da evolução do quadro clínico, o que leva a uma decisão precoce de internação do paciente, e frente a toda a esta situação, observa-se um setor fragmentado desde 0 atendimento inicial, admissão e alta deste paciente (OLIVEIRA LC, et al., 2017; OLIVEIRA LC, et al., 2018).

Sabendo que o SAMU, conforme a Portaria no 3.088/2011 é um dos serviços de atenção às urgências e emergências em saúde mental, compondo a RAPS, em substituição ao atendimento de urgência/emergência nos hospitais psiquiátricos. Entretanto na maioria das vezes, os pacientes em crise psíquica são encaminhados para internação hospitalar, priorizando sedação destes, como sendo a principal e única possibilidade para conter a crise (BONFADA e GUIMARÃES, 2015; OLIVEIRA e SILVA, 2017; SOUZA AS, et al., 2017). A pesquisa de Veloso $C$, et al. (2018) contribui que a assistência ofertada no aspecto das crises psíquicas, apresenta deficiência, bem como um acolhimento inadequado por parte dos profissionais. 
A elaboração e implementação de protocolos específicos voltados para as urgências e emergências psiquiátricas, seria uma alternativa viável, visto que, os atendimentos são baseados em protocolos gerais do SAMU, pois para este público deve existir uma forma mais equânime no que diz respeito às suas necessidades (OLIVEIRA LC, et al., 2017; OLIVEIRA LC, et al., 2018).

Deste modo, pode-se dizer que é preciso de medidas mais rigorosas, que possam atender à necessidade das pessoas que vivem em meio a esta vulnerabilidade, que estão as margens da sociedade, ainda esquecidos e vistos como "loucos", dispondo de diversos direitos que foram adquiridos com muitas perdas por décadas, direitos esses que não são postos em práticas em muitas das vezes, cabendo-nos o dever de contribuir com a saúde mental, e a melhora no padrão de qualidade dos atendimentos diários destas pessoas.

\section{A formação e a experiência profissional}

Passados 40 anos dos movimentos pela reforma psiquiátrica no Brasil, muitos profissionais de saúde, encontram-se presos no passado manicomial, bem como, a falta de preparo para atender as pessoas com transtornos mentais. Tais concepções interferem no cuidado e na compreensão de como atuar em situações de emergências psiquiátricas, sendo que, nem toda crise é sinônimo de uma intervenção, no que diz respeito às contenções, isso demonstra a incapacidade de compreender e aceitar o outro (BRITO AAC, et al., 2015; ANDRADE ACS, et al., 2016).

Sendo assim, quanto menor for a experiência, conhecimento e aceitação dos profissionais em relação as pessoas com algum tipo de transtorno mental, que necessitem de ajuda e acolhimento nas mais diversas situações, dificulta um atendimento e a captação destes para a realização de um tratamento adequado, visto que, para a equipe do SAMU, a tomada de decisão baseia-se em seu tempo de atuação, conhecimentos, e na prática deste tipo de ocorrência no atendimento pré-hospitalar (ANDRADE ACS, et al., 2016).

De acordo Brito ACC, et al. (2015) os profissionais do SAMU precisam compreender quando de fato precisem de apoio ou de medidas mais agressivas para conter um caso de urgência psiquiátrica, pois ainda existe muito preconceito envolvido, falta de capacitação e equidade. Para Brolese DF, et al. (2017) a vivência profissional na atuação em saúde mental, resulta em um maior aprendizado e estimula a qualificação, pois muitos têm a dificuldade de correlacionar a teoria com a prática ao longo da formação.

Percebe-se que, existe uma deficiência de enfermeiros com especializações relacionadas a enfermagem psiquiátrica, existindo uma privação do conhecimento quando equiparadas as demais áreas, sendo que uma pós-graduação na área da psiquiatria contribui para um melhor preparo na atuação. O estigma do preconceito ou a falta de incentivo, influencia na escolha da especialização, fazendo com que enfermeiros optem por outras áreas de atuação (VARGAS D, et al., 2017).

Diante dessas potencialidades, a assistência de uma equipe de urgência e emergência se torna essencial na prestação dos cuidados as pessoas em crise psíquica, visto que ainda existe uma grande dificuldade de identificação dos quadros de emergências psiquiátricas por enfermeiros, podendo levar a uma assistência inadequada, fragmentada e muitas vezes negligenciada (FERNADES MA, et al., 2016; VARGAS D, et al., 2017). Nesta perspectiva, é necessário conhecer de forma ampla como os serviços de urgências emergências psiquiátricas se encontram, compreendendo as necessidades dos profissionais para não gerar julgamentos, podendo desta forma, fornecer o necessário para equipe de saúde, assim como proferir efetividade, eficiência e eficácia dos serviços de saúde (LIMA ICS e GUIMARÃES AB, 2015).

Salienta-se que, se não houverem medidas de integração para com estes pacientes, corre-se o risco de uma atenção desumanizada, perdendo todas as conquistas trazidas pela reforma psiquiátrica, nesta perspectiva a educação permanentes em saúde, demonstra possibilidades de novas mudanças e a valorização da saúde mental por parte dos profissionais nos atendimentos pré-hospitalares, uma vez que não se podem ignorar os aspectos teóricos do pensar sobre o agir, compreender o motivo pelo qual está sendo realizado tal procedimento (SANTOS TO, et al., 2017; OLIVEIRA LC, et al., 2020).

Deste modo, instituir o encorajamento para uma construção de novos modelos de cuidado, atenção e abordagem, exige o entendimento que saúde não deve ser construída apenas no interior dos serviços de saúde, pois é necessário que tenha uma maior compreensão de um cuidado externo, em especifico para as equipes de urgências e emergências psiquiátricas (NÓBREGA MPSS, et al., 2017). 


\section{CONSIDERAÇÕES FINAIS}

Os transtornos psiquiátricos são bastante prevalentes, e as situações de emergências psiquiátricas necessitam de serviços de atendimento que sejam tanto eficazes quanto humanizados. Portanto, a equipe de enfermagem que compõem este departamento precisa compreender o processo dos transtornos mentais e como atuar em diferentes circunstâncias, além da prática é relevante todo conhecimento teórico que possa ser instituída por meio de educação permanente, reiterando-se a importância de pesquisas, protocolos e novos métodos que viabilizem o trabalho dos profissionais de forma a preservar a integridade da pessoa em crise psíquica, pois apesar de contribuírem com a discussão, os achados são limitados na literatura, evidenciando um baixo interesse quanto a esta problemática.

\section{REFERÊNCIAS}

1. ALMEIDA AB, et al. atendimento móvel de urgência na crise psíquica e o paradigma psicossocial. Texto Contexto Enferm, Florianópolis, 2015; 24(4): 1035-43.

2. ANDRADE ACS, et al. Sentimentos de familiares de pacientes internados na emergência psiquiátrica: Um olhar sobre a família. Cienc Cuid Saude 2016; 15(2): 268-274.

3. BASTOS F, et al. Saúde mental no atendimento pré-hospitalar móvel: Concepções de profissionais. Revista Portuguesa de Enfermagem de Saúde Mental, 2016; 18(4): 3-11.

4. BRITO AFG, et al. Atuação do Enfermeiro em Emergências Psiquiátricas. In: Anais da Mostra de Pesquisa em Ciência e Tecnologia 2017; 8:2238-2208.

5. BRITO AAC, et al. Onde a reforma ainda não chegou: ecos da assistência às urgências psiquiátricas. Physis Revista de Saúde Coletiva, Rio de Janeiro, 2015; 1293(1312):20550-013.

6. BROSELE DF, et al. Resilience of the health team in caring for people with mental disorders in a psychiatric hospital. Rev Esc Enferm USP. 2017; 51: e03230.

7. CAVEIRÃO C, et al. Portador de transtorno mental em situação de emergência: dificuldades de atendimento percebidas pela equipe de enfermagem em uma unidade mista Cad. da Esc. de Saúde, Curitiba, 2015; 2(14): 21-31.

8. COSTA JM, et al. A percepção da equipe de enfermagem mediante ás emergências psiquiátricas. Rev Inic Cient Ext [Internet]. 2019; 2(1): 15-23.

9. CRISTIANO CC, et al. Portador de transtorno mental em situação de emergência: Dificuldades de atendimento percebidas pela equipe de enfermagem em uma unidade mista. Cad. da Esc. de Saúde. [Internet] 2015; 2(14): 21-31.

10. DEU-BEM CM, et al. Emergências psiquiátricas: manejo de agitação psicomotora e avaliação de risco suicida, 2017; 50(1):98-112.

11. DUARTE MLC, et al. Percepção dos familiares acerca do grupo de apoio realizado em uma unidade de internação psiquiátrica. Rev Gaúcha Enferm. 2018; 39: e2017-0115.

12. FERNANDES MA, et al. Cuidados de enfermagem ao paciente psiquiátrico na urgência de um hospital geral / Nursing care to psychiatric patients in a general hospital emergency. Rev. enferm. UFPI, 2016; 5(2): 41-45.

13. GOMÇALVEZ KG, et al. Caracterização do atendimento pré-hospitalar às urgências psiquiátricas em um município do interior do estado do Ceará. Revista Nursing, 2019; 22(253): 2932-2936.

14. LIMA ICS e GUIMARÃES A. Perfil das emergências psiquiátricas atendidas em serviços de urgência e emergência hospitalar. R. Interd. 2015; 8(2): 181-190.

15. LOPES MCBT, et al. Avaliação da adesão ao tratamento medicamentoso de pacientes com distúrbios psiquiátricos no serviço de emergência. Enferm. Foco 2018; 19(e565): 2178-2091.

16. MINOLETTI A, et al. Capacidade de resposta da atenção primária à saúde mental no Chile: uma contribuição para AlmaAta. Rev Panam Salud Publica. 2018; 42: e136.

17. NÓBREGA MPSS, et al. A reabilitação psicossocial na rede oeste do município de São Paulo: potencialidades e desafios. Rev Gaúcha Enferm. 2018; 39: e2017-0231.

18. OHARA R, et al. Caracterização do perfil assistencial dos pacientes adultos de um pronto socorro. Rev Bras Enferm [Internet]. 2010; 63(5): 749-54.

19. OLIVEIRA LC, et al. Mobile care service for psychiatric urgencies and emergencies: perception of nursing workers. Rev Bras Enferm. 2020; 73(1): e20180214.

20. OLIVEIRA LC, et al. Saberes e práticas em urgências e emergências psiquiátricas. Rev enferm UERJ, Rio de Janeiro, 2017; 25: e10726.

21. OLIVEIRA LC, et al. Dificuldades no atendimento às urgências e emergências psiquiátricas no serviço de atendimento móvel de urgência. Enferm. Foco 2018; 9(4): 18-22.

22. PAES MR e MAFTUM MA. Communication between nursing team and patients with mental disorder in an emergency service. Cienc Cuid Saúde. [Internet] 2013; 3(3):461-469.

23. PIMENTA FJNA, et al. Ações e práticas de enfermagem frente ao paciente psiquiátrico atendido em um hospital de urgência e emergência de Porto Velho-RO. REAS, 2019; 28: e1059.

24. SOUSA AS, et al. Serviços de atendimento móvel de urgência frente às emergências psiquiátricas: Uma revisão narrativa. Revista Portuguesa de Enfermagem de Saúde Mental, 2018; 72(80): 1647-2160.

25. VARGAS D, et al. Enfermeiros de serviços de urgência e emergência psiquiátrica: análise de perfil profissional e educacional. Cogitare Enferm, 2017; 2(4): e50704.

26. VELOSO C, et al. Atendimentos de natureza psiquiátrica realizados pelo serviço pré-hospitalar móvel de urgência. Texto Contexto Enferm, 2018; 27(2): e0170016. 